OPEN ACCESS

Edited by:

Kai Huang,

University of Texas Medical Branch at Galveston, United States

Reviewed by: Yusuf Tutar,

University of Health Sciences, Turkey Jin-hua He,

Guangzhou Panyu Central Hospital,

China

${ }^{*}$ Correspondence:

Yanjun Lu

junyanlu_2000@163.com

Liming Cheng

chengliming2015@163.com

${ }^{+}$These authors have contributed equally to this work

Specialty section:

This article was submitted to Molecular and Cellular Oncology,

a section of the journal

Frontiers in Oncology

Received: 22 July 2020 Accepted: 30 December 2020 Published: 11 February 2021

Citation:

Wang X, Li H, Lu Y and Cheng L (2021) Regulatory Effects of Circular RNAs on Host Genes in Human Cancer.

Front. Oncol. 10:586163. doi: 10.3389/fonc.2020.586163

\section{Regulatory Effects of Circular RNAs on Host Genes in Human Cancer}

\author{
Xiong Wang ${ }^{\dagger}$, Huijun $\mathrm{Li}^{\dagger}$, Yanjun Lu ${ }^{*}$ and Liming Cheng ${ }^{*}$ \\ Department of Laboratory Medicine, Tongji Hospital, Tongji Medical College of Huazhong University of Science and \\ Technology, Wuhan, China
}

Circular RNAs (circRNAs) are a class of single-stranded, covalent closed-loop RNAs with tissue-/development-specific expression patterns. circRNAs are stable and play oncogenic or tumor suppressive roles in various aspects of cancer, including tumorigenesis, proliferation, apoptosis, metastasis, invasion, chemo-therapeutic resistance, and prognosis. circRNAs act as miRNA/protein sponges, protein scaffold, or template for translation. Increasing evidence shows circRNAs contribute to cancer progression via modulating the expression or function of their host genes. In this review, we summarize the latest progress in the regulation of host genes by circRNAs in human cancer. The works on circRNAs mediated regulation of host genes enhance us to understand the interaction between circRNAs and their host genes in human cancer.

Keywords: circRNA, host gene, transcription, miRNA sponge, cancer

\section{INTRODUCTION}

Circular RNAs (circRNAs) have a history of nearly 30 years, initially considered as the outcome of transcriptional noise. circRNAs are a special class of noncoding RNAs (ncRNAs) derived from the back-splicing of pre-mRNAs or long non-coding RNAs (lncRNAs), and are more stable than linear host genes $(1,2)$. With the advances of deep sequencing and bioinformatics tools, a rapid increasing number of circRNAs have been identified in eukaryotic cells, and the expression profiles of circRNAs are tissue-/development-specific (3).

circRNAs gain increasing attention in the field of human cancer. The functions of circRNAs include adsorbing microRNAs (miRNAs) or proteins as sponges, regulating transcription or alternative splicing, interacting with RNA-binding proteins (RBPs), and translation $(4,5)$. Dysregulated circRNAs play oncogenic or tumor suppressive roles in various aspects of cancer, including cancer initiation, proliferation, apoptosis, metastasis, invasion, chemo-therapeutic resistance, and prognosis $(6,7)$. circRNAs may be diagnostic or therapeutic targets in human cancer $(8,9)$.

Accumulating evidence shows that circRNAs contribute to cancer progression via positively or negatively modulating the expression and function of their host genes $(10-12)$. In this review, we

\footnotetext{
Abbreviations: circRNA, circular RNA; lncRNA, long non-coding RNA; miRNA, microRNA; RBP, RNA-binding protein; 3'UTR, 3'-untranslated region; MRE, miRNA response element; TNBC, triple negative breast cancer; LUAD, lung adenocarcinoma; EMT, epithelial-mesenchymal transition; GC, gastric cancer; HCC, hepatocellular carcinoma; LSCC, laryngeal squamous cell carcinoma; ESCC, esophageal squamous cell carcinoma; OSCC, oral squamous cell carcinoma; PC, prostate cancer; RIP, RNA immunoprecipitation.
} 
summarize the latest progress in circRNA research on circRNA mediated regulation of host genes in human cancer.

\section{REGULATORY EFFECTS OF CIRCRNAS ON HOST GENES IN HUMAN CANCER}

Back-splicing occurs coupled with the splicing of linear host RNAs, and may affect the splicing of linear RNAs (13). However, the efficiency of back-splicing is less than $1 \%$ of the canonical splicing (14). circRNAs can modulate the expression and function of their host genes via acting as miRNA sponges, regulating transcription, stability of mRNA, protein activity, and degradation. Moreover, circRNAs can regulate the expression of host genes by diverse regulatory effects or indirect pathways in human cancer.

\section{circRNAs Regulate Host Gene Expression as miRNA Sponges}

circRNAs contain miRNA response elements (MREs) which bind to miRNAs and restrain their binding to the target mRNAs. Acting as miRNA sponges is the most extensively studied functions of circRNAs. Several circRNAs act as oncogenes in human cancer involving proliferation, migration, invasion, metastasis, glycolysis, and apoptosis by regulating the expression of their host genes via acting as miRNA sponges. Chen et al. found that circFNTA (hsa_circ_0084171), containing exon 2 to 6 of the FNTA gene, was increased in bladder cancer tissues from 41 patients as compared with adjacent para-tumor tissues. circFNTA promoted bladder cancer metastases and reduced cisplatin chemo-sensitivity by sponging miR-370, and the host gene of circFNTA FNTA was the downstream target of miR-370. Their work indicates that circRNAs may act as miRNA sponge to regulate the expression of their host genes, leading to bladder cancer metastasis and chemo-resistance (15). He et al. applied circRNA microarray and qPCR to screen and validate differently expressed circRNAs in triple negative breast cancer (TNBC) cell lines. circGFRA1 (hsa_circ_0005239) was increased in both TNBC cell lines and patients. High expression of
circGFRA1 was correlated with poorer survival in 222 TNBC patients. circGFRA1 promoted proliferation and inhibited apoptosis by binding to miR-34a, relieving GFRA1 (16). Lung adenocarcinoma (LUAD) is one of the predominant reasons of cancer-related mortality. Enolase 1 (ENO1) is a glycolysis enzyme for glucose metabolism, and contributes to tumor progression. Zhou et al. carried out circRNA sequencing to identify differently expressed circRNAs in LUAD, and circENO1 (hsa_circ_0000013) was validated to be upregulated in LUAD tissues and cell lines. Mechanistically, circENO1 induced glycolysis of LUAD cells by acting as competing endogenous RNA (ceRNA) of miR-22-3p to promote the expression of ENO1 (17).

On the other hand, several circRNAs act as tumor suppressors in human cancer via regulating the expression of their host genes through sponging miRNAs. Lv et al. applied RNA sequencing and qPCR to validate that circEPB41L5 was decreased in 49 glioblastoma tissues compared with six normal brain tissues. Low expression of circEPB41L5 was correlated to poor overall survival (OS) and progression-free survival (PFS). circEPB41L5 was derived from exons 17-25 of the EPB41L5 gene. In vitro and in vivo experiments showed that circEPB41L5 inhibited proliferation, migration, clone formation, and invasion of glioblastoma by targeting the miR-19a/EPB41L5 axis (18).

These studies revealed that circRNAs may act as oncogene or tumor suppressor to regulate cancer growth, metabolism, apoptosis, metastasis, and drug-resistance by sponging miRNAs to release or inhibit the expression of their host genes (Table 1).

\section{circRNAs Regulate the Transcription of Host Genes}

circRNAs are able to regulate transcription and splicing in human cancer by recruiting proteins to the specific regions of target genes or sponging proteins to prevent their binding. Proteins include RBPs, transcription factors, DNA demethylase, and DNA methyltransferase. Promoter regions are the most widely studied specific regions for transcription regulation. circRNAs can positively or negatively regulate host gene transcription. Feng et al. screened circRNAs involved in prostate cancer (PC) progression using

TABLE 1 | circRNAs regulate host gene expression as miRNA sponges in human cancer.

\begin{tabular}{lllll}
\hline circRNA & Cancer & Change & miRNA & Effect \\
\hline circFNTA (hsa_circ_0084171) & Bladder cancer & up & miR-370-3p & oncogenic \\
circVANGL1 & Bladder cancer & up & miR-605-3p & oncogenic \\
circITCH & Breast cancer & down & miR-7/-214 & suppressive \\
circGFRA1 (hsa_circ_0005239) & Breast cancer & up & (15) \\
CircAMOTL1 (hsa_circ_0004214) & Cervical cancer & up & miR-34a & oncogenic \\
circEPB41L5 & Glioblastoma & down & miR-485-5p & oncogenic \\
circITCH & Glioma & down & miR-19a & suppressive \\
circSMO742 & Glioma & up & miR-214 & suppressive \\
circFLNA (hsa_circ_0092012) & LSCC & up & miR-338-3p & oncogenic \\
circENO1 (hsa_circ_0000013) & LUAD & up & miR-486-3p & oncogenic \\
circITCH & Lung cancer & down & miR-22-3p & oncogenic \\
CircLARP4 & Ovarian cancer & down & miR-7/-214 & suppressive \\
\hline
\end{tabular}

LSCC, laryngeal squamous cell carcinoma; LUAD, lung adenocarcinoma. 
circRNA microarray. circXIAP (hsa_circ_0005276), derived from three exons of XIAP, and its host gene XIAP were both upregulated in PC. circXIAP was located in both cytoplasm and nucleus. circXIAP recruited FUS binding protein (FUS) to the promoter region of XIAP gene in nucleus and activated the transcription of $X I A P$ so as to promote the proliferation, migration, and epithelialmesenchymal transition (EMT) of PC cells (27). FECR1 was a novel circRNA consisting of FLI1 exons 4-2-3, bound to the promoter of FLI1 and recruited DNA demethylase TET1 to induce DNA demethylation, increasing FLI1 expression and promoting invasion of breast cancer cells (11). circCUX1 (hsa_circ_0132813), consisting of exon 2 and partial intron 2 of CUX1, was localized in the nucleus and increased in neuroblastoma. circCUX1 interacted with EWS RNA-binding protein 1 (EWSR1) and facilitated EWSR1-mediated transactivation of MYC-associated zinc finger protein (MAZ), promoting the transcriptional of CUX1, glycolysis and neuroblastoma progression (28).

circRNAs may also suppress cancer progression via impairing the transcription of their host genes through sponging RBP. circHuR (hsa_circ_0049027), containing exon 3 to 5 of $\mathrm{HuR}$, was predominantly localized in the nucleus, and was decreased in gastric cancer (GC). circHuR interacted with CCHC-type zinc finger nucleic acid binding protein (CNBP), and restrained its binding to the promoter region of $\mathrm{HuR}$, resulting in transcriptional repression of $H u R$ and GC progression (29).

Exonic circRNAs (EcRNAs) were considered to mainly localize in the cytoplasm (30), while intronic circRNAs (CiRNAs) predominantly functioned in the nucleus (31). However, these mentioned EcRNAs were also distributed in the nucleus and regulated the transcription of their host genes $(11,27,28)$. These recent studies suggest that EcRNAs not only functioned in cytoplasm, but also play pivotal roles in nucleus including regulating the transcription of their host genes (Table 2 ).

\section{circRNAs Regulate the mRNA Stability of Host Genes}

Beside of transcriptional regulation of host genes, circRNAs can also regulate the expression of host genes post-transcriptionally through affecting mRNA stability. circRNAs regulate the stability of host gene mRNA via recruiting or sponging RBPs which could bind with mRNA to enhance mRNA stability or induce mRNA instability. circE2F3 (hsa_circ_0075804), localized in cytoplasm, was upregulated in retinoblastoma, and overexpression of circE2F3 promoted the proliferation of retinoblastoma by increasing E2F3 expression. Heterogeneous nuclear ribonucleoprotein $\mathrm{K}$ (HNRNPK) was able to bind with target mRNA and enhance mRNA stability. circE2F3 bound with HNRNPK and facilitate HNRNPK-mediated mRNA stability of E2F3, improving the stability of E2F3 mRNA in retinoblastoma (32).

On the other hand, circRNAs may also induce mRNA instability of their host genes to suppress the expression of host genes. HuR is an extensively studied RBP which is able to bind with a wide range of RNAs, leading to translational promotion or suppression of target mRNAs through elongating or shortening the half-life of target mRNAs. By using RNA immunoprecipitation (RIP) and circRNA microarray, Abdelmohsen et al. screened HuR-interacted circRNAs in HeLa cells. circPABPN1 (hsa_circ_0031288) was the most highly enriched HuR-interacted circRNA. circPABPN1 competitively bound with HuR to disrupt the binding between HuR and PABPN1 mRNA, leading to instability of PABPN1 mRNA and translational suppression of its host gene in Hela cells (33).

The mentioned circRNAs mainly regulated the translation of host genes in cytoplasm. Interestingly, circRNAs may play this regulatory role in nucleus. AUF1 is an AU-rich RBP which can shuttle between cytoplasm and nucleus. AUF1 could bind to the $3^{\prime}$-untranslated region (3'-UTR) of DNMT1 mRNA and promote its degradation. circDNMT1 interacted with AUF1 and facilitated the nuclear translocation of AUF1, relieving DNMT1 mRNA from AUF1 induced instability of DNMT1 mRNA in breast cancer (34).

By binding with RBPs which are able to promote or suppress mRNA degradation, circRNAs may regulate the expression of their host genes in both cytoplasm and nucleus (Table 3).

TABLE 2 | circRNAs regulate host gene transcription in human cancer.

\begin{tabular}{|c|c|c|c|c|c|c|}
\hline circRNA & Cancer & Change & Effect on host gene & Protein-interaction & Effect on cancer & Ref \\
\hline circXIAP (hsa_circ_0005276) & Prostate cancer & up & promotive & FUS & oncogenic & $(27)$ \\
\hline FECR1 & Breast cancer & - & promotive & TET1 & oncogenic & (11) \\
\hline circCUX1 (hsa_circ_0132813) & Neuroblastoma & up & promotive & EWSR1 & oncogenic & (28) \\
\hline circHuR (hsa_circ_0049027) & Gastric cancer & down & suppressive & CNBP & suppressive & (29) \\
\hline
\end{tabular}

TABLE 3 | circRNAs regulate the stability of host gene mRNA in human cancer.

\begin{tabular}{|c|c|c|c|c|c|c|}
\hline circRNA & Cancer & Change & Effect on host gene & Protein-interaction & Effect on cancer & Ref \\
\hline circE2F3 (hsa_circ_0075804) & Retinoblastoma & up & promotive & HNRNPK & oncogenic & $(32)$ \\
\hline circDNMT1 & Breast cancer & up & promotive & AUF1 & oncogenic & (34) \\
\hline circPABPN1 (hsa_circ_0031288) & Cervical cancer & - & suppressive & HuR & suppressive & (33) \\
\hline
\end{tabular}




\section{circRNAs Regulate the Activity and Degradation of Parental Proteins}

Post-translational modifications are essential for the activity and degradation of proteins, such as acetylation, ubiquitination, sumoylation, and peptidyl-prolyl isomerization. Wnt signaling pathway contributes to the carcinogenesis of many malignancies, and GSK $3 \beta$ is a negative mediator of this pathway by phosphorylating Wnt and inducing ubiquitination-mediated degradation of Wnt. Hu et al. found that $\operatorname{circGSK} 3 \beta$ (hsa_circ_0007986) was localized in the cytoplasm and increased in esophageal squamous cell carcinoma (ESCC). Increased circGSK3 $\beta$ was positively correlated with advanced clinical stage and poor patient prognosis of ESCC. By using RIP and mass spectrometry, GSK $3 \beta$, the parental protein of circGSK $3 \beta$, was found to bind with circGSK3 $\beta$ in ESCC cell lines. circGSK3 $\beta$ bound to the N-terminal of GSK3 $\beta$ directly and inhibited its phosphorylation on Wnt, augmenting $\beta$-catenin signaling in ESCC (35). MDM2 is an E3 ubiquitin ligase and promotes tumor progression by targeting tumor suppressor proteins for ubiquitination mediated degradation, such as $\mathrm{p} 53$. $\mathrm{Du}$ et al. found that circFOXO3 acted as a protein scaffold recruiting both MDM2 and p53 to form MDM2-p53 complex via binding with the RING-finger domain of MDM2 and Cterminal regulatory domain of $\mathrm{p} 53$ respectively. circFOXO3 induced MDM2-mediated ubiquitination and degradation of p53 released FOXO3 from MDM2 induced ubiquitination and degradation, promoting the progression of breast cancer (36). These studies suggest that circRNAs may regulate the activity and degradation of parental protein by direct interacting with parental protein or disrupting the post-translational modification and subsequential degradation.

\section{Diverse Regulatory Effects of circRNAs on Host Gene in Human Cancer}

circRNAs could regulate the expression of host genes via diverse pathways simultaneously. circCCND1 (hsa_circ_0023303) was increased in LSCC, and closely associated with adverse prognosis. circCCND1 bound to $\mathrm{HuR}$ and enhanced the binding of HuR to the $3^{\prime}$-UTR of CCND1 mRNA to stabilize CCND1 mRNA. On the other hand, circCCND1 sponged miR646 to release CCND1. circCCND1 promoted the progression of LSCC through increasing CCND1 expression by increasing its mRNA stability and disrupting miR-646 mediated suppression (37). AUF1 could bind to the $3^{\prime}$-UTR of target mRNA and induce mRNA degradation (34). circMMP9 (hsa_circ_ 0001162) was upregulated in oral squamous cell carcinoma (OSCC), and positively correlated with advanced TNM stage. circMMP9 simultaneously interacted with AUF1 and miR-149 to alleviate their inhibitory effect on MMP9 3'-UTR, resulting in enhanced MMP9 expression and facilitating OSCC metastasis (38). circFBXW7 (hsa_circ_0001451) was decreased in TNBC, and was able to encode a novel $185 \mathrm{aa}$ peptide named FBXW7-185aa. FBXW7-185aa peptide bound to the deubiquitinating enzyme USP28 and prevented USP28 from binding to FBXW7, increasing the abundance of FBXW7. Moreover, circFBXW7 increased FBXW7 expression via serving as a sponge of miR-197-3p to inhibit malignant progression of TNBC (39).

circRNAs may also regulate host gene expression via different mechanisms in different types of cancer. circSHPRH (hsa_circ_0001649) was decreased in both glioblastoma and hepatocellular carcinoma (HCC). circSHPRH inhibited HCC progression via acting as a ceRNA to sponge miR-127-5p, miR612 and $\mathrm{miR}-4688$ to relieve SHPRH (40). In glioblastoma, circSHPRH encoded a peptide named SHPRH-146aa. SHPRH146aa competitively interacted with E3 ligase DTL, protecting fulllength SHPRH from ubiquitination by DTL. Full-length SHPRH then suppressed glioblastoma progression by inducing proliferating cell nuclear antigen (PCNA) ubiquitination as an E3 ligase (10).

These studies support the cancer specific and complicated regulation of circRNAs on their host genes. This regulation of host genes by circRNAs may involve diverse mechanisms of circRNAs and may also be different in different types of cancer (Table 4).

\section{CONCLUSION}

A number of host genes of circRNAs play oncogenic or suppressive role in tumorigenesis. circRNAs are derived from these genes and regulate the transcription, translation, parental protein activity and degradation of their host genes. Similar with the regulatory effects of circRNAs on other targets, circRNAs regulate host gene expression mainly via acting as miRNA sponges in human cancer. In addition, circRNAs may recruit or sponge some proteins to enhance or retard the transcription and mRNA stability of host genes. circRNAs

TABLE 4 | Diverse regulatory effects of circRNAs on host gene in human cancer.

\begin{tabular}{|c|c|c|c|c|c|c|c|}
\hline circRNA & Cancer & Change & Effect on host gene & Protein-interaction & Pathway & Effect on cancer & Ref \\
\hline circCCND1 (hsa_circ_0023303) & LSCC & up & promotive & HuR & $\begin{array}{l}\text { circCCND1/HuR/CCND1 } \\
\text { circCCND1/miR-646/CCND1 }\end{array}$ & oncogenic & $(37)$ \\
\hline circMMP9 (hsa_circ_0001162) & OSCC & up & promotive & AUF1 & $\begin{array}{l}\text { circMMP9/miR-149/MMP9 } \\
\text { circMMP/AUF1/MMP9 }\end{array}$ & oncogenic & $(38)$ \\
\hline circFBXW7 (hsa_circ_0001451) & Breast cancer & down & promotive & USP28 & $\begin{array}{l}\text { circFBXW7/miR-197-3p/FBXW7 } \\
\text { circFBXW7/peptide/USP28/FBXW7 }\end{array}$ & suppressive & (39) \\
\hline
\end{tabular}

OSCC, oral squamous cell carcinoma. 
can directly bind with host protein as protein sponge, or recruit proteins to regulate post-translational modification such as ubiquitination. Moreover, the regulation of host genes by circRNAs in cancer may involve a variety of classical mechanisms of circRNAs, and the suppressive mechanisms of circRNAs on host genes may be also different in different types of cancer, indicating the complicated regulatory effects of circRNAs on their host genes. The works on circRNAs mediated regulation of host genes enhance us to understand the interaction between circRNAs and their host genes in human cancer.

\section{REFERENCES}

1. Kristensen LS, Andersen MS, Stagsted LVW, Ebbesen KK, Hansen TB, Kjems J. The biogenesis, biology and characterization of circular RNAs. Nat Rev Genet (2019) 20(11):675-91. doi: 10.1038/s41576-019-0158-7

2. Hanniford D, Ulloa-Morales A, Karz A, Berzoti-Coelho MG, Moubarak RS, Sanchez-Sendra B, et al. Epigenetic Silencing of CDR1as Drives IGF2BP3Mediated Melanoma Invasion and Metastasis. Cancer Cell (2020) 37(1):55-70 e15. doi: 10.1016/j.ccell.2019.12.007

3. Wu W, Ji P, Zhao F. CircAtlas: an integrated resource of one million highly accurate circular RNAs from 1070 vertebrate transcriptomes. Genome Biol (2020) 21(1):101. doi: 10.1186/s13059-020-02018-y

4. Huang A, Zheng $\mathrm{H}$, Wu Z, Chen M, Huang Y. Circular RNA-protein interactions: functions, mechanisms, and identification. Theranostics (2020) 10(8):3503-17. doi: 10.7150/thno.42174

5. Lei M, Zheng G, Ning Q, Zheng J, Dong D. Translation and functional roles of circular RNAs in human cancer. Mol Cancer (2020) 19(1):30. doi: 10.1186/ s12943-020-1135-7

6. Li J, Sun D, Pu W, Wang J, Peng Y. Circular RNAs in Cancer: Biogenesis, Function, and Clinical Significance. Trends Cancer (2020) 6(4):319-36. doi: 10.1016/j.trecan.2020.01.012

7. Wu J, Qi X, Liu L, Hu X, Liu J, Yang J, et al. Emerging Epigenetic Regulation of Circular RNAs in Human Cancer. Mol Ther Nucleic Acids (2019) 16:58996. doi: 10.1016/j.omtn.2019.04.011

8. Sun J, Li B, Shu C, Ma Q, Wang J. Functions and clinical significance of circular RNAs in glioma. Mol Cancer (2020) 19(1):34. doi: 10.1186/s12943019-1121-0

9. Shan C, Zhang Y, Hao X, Gao J, Chen X, Wang K. Biogenesis, functions and clinical significance of circRNAs in gastric cancer. Mol Cancer (2019) 18 (1):136. doi: 10.1186/s12943-019-1069-0

10. Zhang M, Huang N, Yang X, Luo J, Yan S, Xiao F, et al. A novel protein encoded by the circular form of the SHPRH gene suppresses glioma tumorigenesis. Oncogene (2018) 37(13):1805-14. doi: 10.1038/s41388-0170019-9

11. Chen N, Zhao G, Yan X, Lv Z, Yin H, Zhang S, et al. A novel FLI1 exonic circular RNA promotes metastasis in breast cancer by coordinately regulating TET1 and DNMT1. Genome Biol (2018) 19(1):218. doi: 10.1186/s13059-018-1594-y

12. Yang F, Fang E, Mei H, Chen Y, Li H, Li D, et al. Cis-Acting circ-CTNNB1 Promotes beta-Catenin Signaling and Cancer Progression via DDX3Mediated Transactivation of YY1. Cancer Res (2019) 79(3):557-71. doi: 10.1158/0008-5472.CAN-18-1559

13. Ashwal-Fluss R, Meyer M, Pamudurti NR, Ivanov A, Bartok O, Hanan M, et al. circRNA biogenesis competes with pre-mRNA splicing. Mol Cell (2014) 56(1):55-66. doi: 10.1016/j.molcel.2014.08.019

14. Zhang Y, Xue W, Li X, Zhang J, Chen S, Zhang JL, et al. The Biogenesis of Nascent Circular RNAs. Cell Rep (2016) 15(3):611-24. doi: 10.1016/ j.celrep.2016.03.058

15. Chen J, Sun Y, Ou Z, Yeh S, Huang CP, You B, et al. Androgen receptorregulated circFNTA activates KRAS signaling to promote bladder cancer invasion. EMBO Rep (2020) 21(4):e48467. doi: 10.15252/embr.201948467

\section{AUTHOR CONTRIBUTIONS}

LC and YL designed this review. XW and HL collected the related paper. XW wrote the manuscript. All authors contributed to the article and approved the submitted version.

\section{FUNDING}

This work was supported by grants from the National Natural Science Foundation of China (81572071 and 81000331).

16. He R, Liu P, Xie X, Zhou Y, Liao Q, Xiong W, et al. circGFRA1 and GFRA1 act as ceRNAs in triple negative breast cancer by regulating miR-34a. J Exp Clin Cancer Res CR (2017) 36(1):145. doi: 10.1186/s13046-017-0614-1

17. Zhou J, Zhang S, Chen Z, He Z, Xu Y, Li Z. CircRNA-ENO1 promoted glycolysis and tumor progression in lung adenocarcinoma through upregulating its host gene ENO1. Cell Death Dis (2019) 10(12):885. doi: 10.1038/s41419-019-2127-7

18. Lv T, Miao Y, Xu T, Sun W, Sang Y, Jia F, et al. Circ-EPB41L5 regulates the host gene EPB41L5 via sponging miR-19a to repress glioblastoma tumorigenesis. Aging (2020) 12(1):318-39. doi: 10.18632/aging.102617

19. Zeng Z, Zhou W, Duan L, Zhang J, Lu X, Jin L, et al. Circular RNA circVANGL1 as a competing endogenous RNA contributes to bladder cancer progression by regulating miR-605-3p/VANGL1 pathway. J Cell Physiol (2019) 234(4):3887-96. doi: 10.1002/jcp.27162

20. Wang ST, Liu LB, Li XM, Wang YF, Xie PJ, Li Q, et al. Circ-ITCH regulates triple-negative breast cancer progression through the Wnt/beta-catenin pathway. Neoplasma (2019) 66(2):232-9. doi: 10.4149/neo_2018_180710N460

21. Ou R, Lv J, Zhang Q, Lin F, Zhu L, Huang F, et al. circAMOTL1 Motivates AMOTL1 Expression to Facilitate Cervical Cancer Growth. Mol Ther Nucleic Acids (2020) 19:50-60. doi: 10.1016/j.omtn.2019.09.022

22. Li F, Ma K, Sun M, Shi S. Identification of the tumor-suppressive function of circular RNA ITCH in glioma cells through sponging miR-214 and promoting linear ITCH expression. Am J Trans Res (2018) 10(5):1373-86.

23. Xiong Z, Zhou C, Wang L, Zhu R, Zhong L, Wan D, et al. Circular RNA SMO sponges miR-338-3p to promote the growth of glioma by enhancing the expression of SMO. Aging (2019) 11(24):12345-60. doi: 10.18632/ aging. 102576

24. Wang JX, Liu Y, Jia XJ, Liu SX, Dong JH, Ren XM, et al. Upregulation of circFLNA contributes to laryngeal squamous cell carcinoma migration by circFLNA-miR-486-3p-FLNA axis. Cancer Cell Int (2019) 19:196. doi: 10.1186/s12935-019-0924-9

25. Wan L, Zhang L, Fan K, Cheng ZX, Sun QC, Wang JJ. Circular RNA-ITCH Suppresses Lung Cancer Proliferation via Inhibiting the Wnt/beta-Catenin Pathway. BioMed Res Int (2016) 2016:1579490. doi: 10.1155/2016/1579490

26. Lin W, Ye H, You K, Chen L. Up-regulation of circ_LARP4 suppresses cell proliferation and migration in ovarian cancer by regulating miR-513b-5p/ LARP4 axis. Cancer Cell Int (2020) 20:5. doi: 10.1186/s12935-019-1071-Z

27. Feng Y, Yang Y, Zhao X, Fan Y, Zhou L, Rong J, et al. Circular RNA circ0005276 promotes the proliferation and migration of prostate cancer cells by interacting with FUS to transcriptionally activate XIAP. Cell Death Dis (2019) 10(11):792. doi: 10.1038/s41419-019-2028-9

28. Li H, Yang F, Hu A, Wang X, Fang E, Chen Y, et al. Therapeutic targeting of circ-CUX1/EWSR1/MAZ axis inhibits glycolysis and neuroblastoma progression. EMBO Mol Med (2019) 11(12):e10835. doi: 10.15252/ emmm.201910835

29. Yang F, Hu A, Li D, Wang J, Guo Y, Liu Y, et al. Circ-HuR suppresses HuR expression and gastric cancer progression by inhibiting CNBP transactivation. Mol Cancer (2019) 18(1):158. doi: 10.1186/s12943-019-1094-Z

30. Meng X, Li X, Zhang P, Wang J, Zhou Y, Chen M. Circular RNA: an emerging key player in RNA world. Briefings Bioinf (2017) 18(4):547-57. doi: 10.1093/ bib/bbw045 
31. Han B, Chao J, Yao H. Circular RNA and its mechanisms in disease: From the bench to the clinic. Pharmacol Ther (2018) 187:31-44. doi: 10.1016/ j.pharmthera.2018.01.010

32. Zhao W, Wang S, Qin T, Wang W, Circular RNA. (circ-0075804) promotes the proliferation of retinoblastoma via combining heterogeneous nuclear ribonucleoprotein $\mathrm{K}$ (HNRNPK) to improve the stability of E2F transcription factor 3 E2F3. J Cell Biochem (2020) 121(7):3516-25. doi: $10.1002 /$ jcb. 29631

33. Abdelmohsen K, Panda AC, Munk R, Grammatikakis I, Dudekula DB, De S, et al. Identification of HuR target circular RNAs uncovers suppression of PABPN1 translation by CircPABPN1. RNA Biol (2017) 14(3):361-9. doi: 10.1080/15476286.2017.1279788

34. Du WW, Yang W, Li X, Awan FM, Yang Z, Fang L, et al. A circular RNA circDNMT1 enhances breast cancer progression by activating autophagy. Oncogene (2018) 37(44):5829-42. doi: 10.1038/s41388-018-0369-y

35. Hu X, Wu D, He X, Zhao H, He Z, Lin J, et al. circGSK3beta promotes metastasis in esophageal squamous cell carcinoma by augmenting betacatenin signaling. Mol Cancer (2019) 18(1):160. doi: 10.1186/s12943-0191095-y

36. Du WW, Fang L, Yang W, Wu N, Awan FM, Yang Z, et al. Induction of tumor apoptosis through a circular RNA enhancing Foxo3 activity. Cell Death Diff (2017) 24(2):357-70. doi: 10.1038/cdd.2016.133

37. Zang Y, Li J, Wan B, Tai Y. circRNA circ-CCND1 promotes the proliferation of laryngeal squamous cell carcinoma through elevating CCND1 expression via interacting with HuR and miR-646. J Cell Mol Med (2020) 24(4):2423-33. doi: $10.1111 /$ jcmm.14925

38. Xia B, Hong T, He X, Hu X, Gao Y. A circular RNA derived from MMP9 facilitates oral squamous cell carcinoma metastasis through regulation of MMP9 mRNA stability. Cell Transplant (2019) 28(12):1614-23. doi: 10.1177/ 0963689719875409

39. Ye F, Gao G, Zou Y, Zheng S, Zhang L, Ou X, et al. circFBXW7 Inhibits Malignant Progression by Sponging miR-197-3p and Encoding a 185-aa Protein in Triple-Negative Breast Cancer. Mol Ther Nucleic Acids (2019) 18:88-98. doi: 10.1016/j.omtn.2019.07.023

40. Su Y, Xu C, Liu Y, Hu Y, Wu H. Circular RNA hsa_circ_0001649 inhibits hepatocellular carcinoma progression via multiple miRNAs sponge. Aging (2019) 11(10):3362-75. doi: 10.18632/aging.101988

Conflict of Interest: The authors declare that the research was conducted in the absence of any commercial or financial relationships that could be construed as a potential conflict of interest.

Copyright (c) 2021 Wang, Li, Lu and Cheng. This is an open-access article distributed under the terms of the Creative Commons Attribution License (CC BY). The use, distribution or reproduction in other forums is permitted, provided the original author(s) and the copyright owner(s) are credited and that the original publication in this journal is cited, in accordance with accepted academic practice. No use, distribution or reproduction is permitted which does not comply with these terms. 